\title{
DNA Polymerase: Structural Homology, Conformational Dynamics, and the Effects of Carcinogenic DNA Adducts
}

\author{
Richard G. Federley and Louis J. Romano \\ Department of Chemistry, Wayne State University, Detroit, MI 48202, USA \\ Correspondence should be addressed to Louis J. Romano, ljr@chem.wayne.edu
}

Received 14 May 2010; Accepted 30 June 2010

Academic Editor: Ashis Basu

Copyright $\odot 2010$ R. G. Federley and L. J. Romano. This is an open access article distributed under the Creative Commons Attribution License, which permits unrestricted use, distribution, and reproduction in any medium, provided the original work is properly cited.

\begin{abstract}
DNA replication is vital for an organism to proliferate and lying at the heart of this process is the enzyme DNA polymerase. Most DNA polymerases have a similar three dimensional fold, akin to a human right hand, despite differences in sequence homology. This structural homology would predict a relatively unvarying mechanism for DNA synthesis yet various polymerases exhibit markedly different properties on similar substrates, indicative of each type of polymerase being prescribed to a specific role in DNA replication. Several key conformational steps, discrete states, and structural moieties have been identified that contribute to the array of properties the polymerases exhibit. The ability of carcinogenic adducts to interfere with conformational processes by directly interacting with the protein explicates the mutagenic consequences these adducts impose. Recent studies have identified novel states that have been hypothesised to test the fit of the nascent base pair, and have also shown the enzyme to possess a lively quality by continually sampling various conformations. This review focuses on the homologous structural changes that take place in various DNA polymerases, both replicative and those involved in adduct bypass, the role these changes play in selection of a correct substrate, and how the presence of bulky carcinogenic adducts affects these changes.
\end{abstract}

\section{Introduction}

Accurate replication of genomic DNA is imperative for the successful proliferation of an organism. The enzyme DNA polymerase is responsible for catalyzing the templatedirected addition of a deoxyribonucleoside- $5^{\prime}$-triphosphate $\left(\mathrm{dNTP}^{1}\right)$ to a growing DNA strand and must do so rapidly and with high fidelity. It is known that the thermodynamics of base pairing alone are insufficient to account for the incredibly low error rates achieved during DNA synthesis [1], and that the DNA polymerase imposes constraints on the selection of a correct nucleotide for incorporation into the primer. Utilizing the thermodynamics of base pairing alone can only account for an accuracy of 1 mistake in 150 nucleotides incorporated, whereas polymerases are known to have error frequencies ranging from $10^{-3}$ to $10^{-5}$ $[2,3]$. DNA polymerases must not only ensure accurate synthesis through selection of a correct dNTP, but they also must prevent the incorporation of incorrect substrates such as ribonucleoside- $5^{\prime}$-triphophates (rNTPs). Therefore, a stringent mechanism must be in place for rejection of correctly base pairing nucleotides having a ribose $2^{\prime}$ hydroxyl. In addition, some DNA polymerases must cope with reading templates containing DNA adducts that have evaded nucleotide or base excision repair. These roadblocks to replication, such as the well studied DNA adducts 2aminofluorine (AF) and $N$-acetyl-2-aminofluorene (AAF), present a tough challenge to the polymerase by altering the template structure, often causing a misincorporation event to occur, the generation of a frameshift, or simply place a stringent block to the advancement of replication $[4,5]$.

Normally, DNA replication proceeds via nucleotide synthesis performed by replicative polymerases. These polymerases are efficient at incorporating bases that allow proper Watson-Crick hydrogen bonding and also fulfil strict geometric constraints within the polymerase active site. However, the replication past the aforementioned DNA adducts presents a special problem to the tight constraints of a replicative polymerase's active site. To alleviate these roadblocks, a special class of polymerases exist to fill such a 
niche. The bypass or translesion synthesis (TLS) polymerases have the ability to accommodate a vast array of DNA lesions and perform either incorporation or extension in regions where replicative polymerases stall or fail completely. This paper will focus on the homologous conformational changes that take place in various DNA polymerases, the role these changes play in selection of a correct substrate, and how the presence of bulky carcinogenic adducts affects these changes.

\section{A Face for a Name}

E. coli DNA polymerase I plays a role in the repair of damaged duplex DNA and the processing of Okazaki fragments in E. coli [3]. This polymerase consists of a multidomain architecture housing not only the $5^{\prime}-3^{\prime}$ polymerization activity required for DNA replication, but also $3^{\prime}-5^{\prime}$ and $5^{\prime}-3^{\prime}$ exonuclease activities. The $3^{\prime}-5^{\prime}$ exonuclease, or proofreading activity, allows greater fidelity to be achieved by removing incorrectly incorporated nucleotides from the growing DNA primer strand resulting in another opportunity to incorporate the correct base before continuing on with synthesis. The $5^{\prime}-3^{\prime}$ exonuclease does not directly function in increasing fidelity but instead is involved with processing of Okazaki fragments by the excision of RNA primers situated on the lagging strand.

A very useful proteolytic digest of DNA polymerase I produces a truncated enzyme termed Klenow fragment. Klenow fragment houses the $5^{\prime}-3^{\prime}$ polymerase activity required for DNA synthesis and the $3^{\prime}-5^{\prime}$ exonuclease proofreading activity, but is devoid of the $5^{\prime}-3^{\prime}$ domain responsible for the excision of RNA primers. In addition, a single D424A point mutation within Klenow fragment's $3^{\prime}-5^{\prime}$ exonuclease domain almost abolishes the exonuclease activity, making this polymerase an excellent choice for DNA synthesis studies without the complication of exonuclease action [9]. Klenow fragment has been a model enzyme for the study of DNA replication for the last four decades, and the solution of the crystal structure in 1985 by Ollis et al. aided in providing a structural overview of the enzymatic domains [10].

The crystal structure of Klenow fragment revealed a two domain architecture, with the smaller 200 amino acid $\mathrm{N}$-terminus forming the $3^{\prime}-5^{\prime}$ exonuclease domain and the larger 400 amino acid C-terminal domain folding to form the polymerization domain. The general shape of the Klenow fragment polymerization domain, being akin to a right hand, has proven to be strikingly universal among DNA polymerases. Klentaq1, the analogous Klenow fragment portion of thermophilic Thermus aquaticus DNA polymerase I, showed a C-terminal fold nearly identical to that of Klenow fragment and can be seen in Figure 1(a) [7]. The polymerase domain can be further divided into three subdomains consisting of the fingers, thumb, and palm, all of which form a cleft of approximately $20-24 \AA$ wide and 25-35 $\AA$ deep [10]. Based on previous biochemical studies identifying catalytically important residues in Klenow fragment, the active site of the C-terminal polymerization domain was mapped to the base of the cleft within the palm of the enzyme, with the $3^{\prime}-5^{\prime}$ exonuclease active site located about $35 \AA$ away. The cleft was noted to be of the approximate size to bind DNA, but it would not be until 1993 when Beese et al. published a cocrystal structure of Klenow fragment bound to duplex DNA that the orientation and structure of the duplex within the enzyme would be known [11].

The cocrystal structure of Klenow bound to duplex DNA gave the first glimpse into the arrangement of the DNA within the polymerase and evidence of the first conformational change that takes place within the enzyme. In this structure, the DNA was situated within the polymerase at right angles to the cleft containing the active polymerase site however, the $3^{\prime}$ primer terminus was melted from the template and situated within the exonuclease site in an editing complex. Despite being in an editing complex, the authors modeled the primer strand at the polymerase active site and concluded that the primer could alternate between the exonuclease and polymerase sites, without dissociation of the enzyme. Interestingly, Beese et al. noted in their modeling that some distortion of the DNA duplex terminus or protein would be required in order to achieve binding at the polymerase site and that this distortion of the DNA would make the equilibrium between single-stranded and doublestranded DNA more sensitive to mismatches. Irrespective of the mode of binding, many important observations were noted from this structure and further confirmed in later crystal structures of Klentaq (Figure 1(b)) [8]. One such observation was the small DNA-induced closing motion the thumb region undergoes upon formation of the binary complex. A region of the thumb, residues 558 to 637 in Klenow fragment, executes a shift ( $12 \AA$ at the $\mathrm{N}$-terminus of helix I) towards the $3^{\prime}-5^{\prime}$ exonuclease domain to form direct contacts with the DNA [11]. Chemical modification studies also showed that Lys635 (Lys540 in Klentaq), which is a highly conserved residue among the Pol I family, is directly involved in DNA binding [12]. In addition to the thumb, extensive contacts are formed between the enzyme and the DNA, although virtually all contacts are nonspecific in nature, forming interactions with the phosphate backbone of the DNA, or to the universal hydrogen bond donors and acceptors of the minor groove. This is important to ensure that the polymerase will bind all DNA equally, irrespective of the sequence. The small closing movement of the thumb upon DNA binding proved to be the first of at least two conformational changes to take place. The initial description of Klenow fragment in terms of a hand, and specifically the naming of the subdomains as fingers and thumb, proved to be somewhat serendipitous due to their anthropomorphic motions upon forming a binary complex, and as was later shown, a catalytically competent ternary complex.

\section{Hold on Tight}

In order to successfully replicate DNA, an active ternary complex must be formed with the proper geometric alignment of the polymerase catalytic residues, the metal ions, the primer-template DNA duplex, and the correctly chosen dNTP. The first crystal structure solutions for the ternary complexes were of Rat DNA polymerase $\beta$, Klentaq1, the 


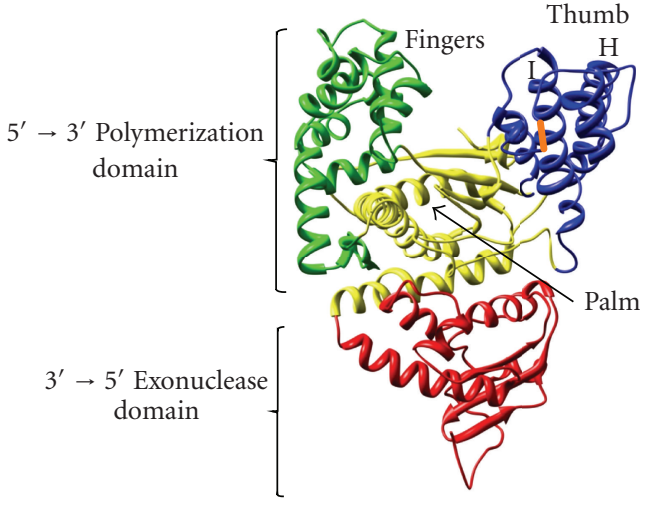

(a)

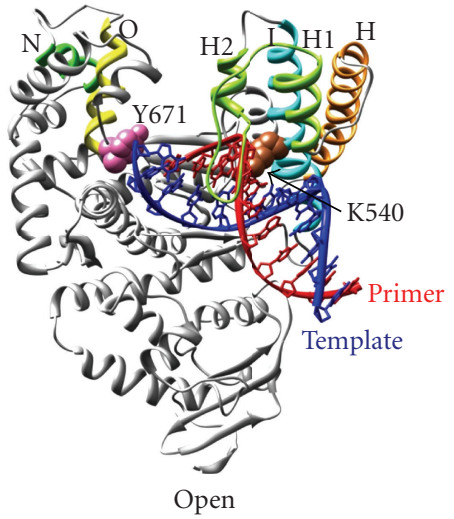

(b)

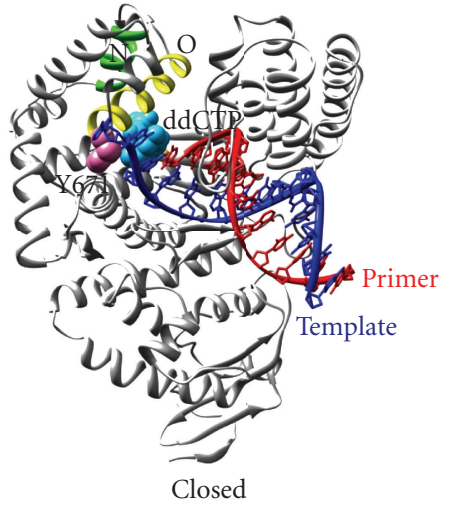

(c)

Figure 1: Structure of Klentaq1 (a) Structure of Klentaq1 in the absence of DNA (PDB \#1KTQ) [7]. The overall structure of polymerases resembles that of a human right hand forming palm (yellow), fingers (green), and thumb (blue) domains. The palm domain houses the active site residues responsible for the $5^{\prime}$ to $3^{\prime}$ polymerase activity, and the $3^{\prime}$ to $5^{\prime}$ exonuclease domain (red) allows excision of misincorporated bases. The H1H2 Loop is disordered and missing from the structure (ends connected with orange line). (b) Klentaq1 open binary complex (PDB \#4KTQ) [8]. The DNA is situated in the cleft formed between the fingers, thumb, and palm domains. The Y671 residue (pink) is stacked on top of the template (dark blue). K540 (brown) makes nonspecific interactions with the minor groove to aid in holding the polymerase bound to the DNA. Helices I (cyan) and H (orange) make small movements relative to their positions in the open complex, and the $\mathrm{H} 1 \mathrm{H} 2$ loop (light green) now becomes ordered and visible in the structure. The remainder of the colors are as follows primer (red), $\mathrm{H} 1$ and H2 helix (light green), O helix (yellow), and N helix (green). (c) Klentaq1 closed ternary complex (PDB \#3KTQ) [8]. A correctly base pairing ddCTP (light blue) is located within the active site inducing a large conformational change of the $\mathrm{O}$ (yellow) and $\mathrm{N}$ (green) helices (compare with positions in (b)). Y671 is now flipped out of its stacking arrangement with the template and the templating base forms Watson-Crick base pairs with the ddCTP. Molecular graphics images were produced using the UCSF Chimera package from the Resource for Biocomputing, Visualization, and Informatics at the University of California, San Francisco (supported by NIH P41 RR-01081) [6].

replicative DNA polymerase of bacteriophage T7, and Bacillus stearothermophilus DNA polymerase I $[8,13-15]$. These structures revealed a great deal of information regarding the location of dNTP binding and catalytically active residues, a large conformational change in the protein, and a two metal ion mechanism for nucleotidyl transfer. Despite the wide breadth of organisms these polymerases were obtained from, all exhibited the now stereotypical polymerase architecture of a human right hand and share a similar geometry of the polymerase active site. What follows is a general culmination of information for most observed structures.

The ternary complex is defined by binding of a dNTP into the polymerase cleft alongside the DNA. The incoming dNTP is aligned within the active site by interactions of the nonbridging oxygens of the phosphate moiety to positively charged residues along and near the $\mathrm{O}$ helix, placing the phosphate moiety roughly parallel to the $\mathrm{O}$ helix. These phosphate interactions are suggested as the primary recognition segment of the incoming $\mathrm{dNTP}$, which is based upon crystal structures of binary complexes of Klentaq 1 with all four dNTPs, showing the phosphates of all four dNTPs aligned in similar positions [16]. However, the base and ribose moieties were in slightly different orientations for the four structures. Most interestingly, the formation of the ternary complex in the presence of the next correct dNTP for base pairing with the first single stranded template base is accompanied by a large conformational change in the fingers of the polymerase (Figure $1(\mathrm{c})$ ). In the case of
Klentaq, this change is an inward rotation of the $\mathrm{O}$ helix by approximately $46^{\circ}\left(41^{\circ}\right.$ in T7) towards the primer-template $[8,14]$. The formation of the closed ternary complex has the effect of clamping down the dNTP into the active site, effectively sealing off the crevice formed by the thumb, palm, and fingers, resulting in the proper geometric alignment of catalytic residues to complete a nucleotidyl transfer reaction.

Further elaboration by Dzantiev and Romano showed that the closed tertiary complex could only be observed in the presence of the next correct nucleotide and not in the presence of incorrectly base pairing nucleotides [17]. Using a tryptic digestion analysis they showed that trypsin was capable of cleaving Klenow fragment near the active site of the polymerization domain in its open form. However, upon incubation of Klenow fragment with a correctly base pairing dNTP the cleavage was inhibited, presumably due to the conformational change of the enzyme protecting the region near the active site from proteolytic cleavage. Further, a correctly base pairing ribonucleotide substrate also did not trigger this conformational change as evidence by the formation of trypsin cleavage products. This suggested that only a correctly base pairing dNTP could conform to the close fitting active site of the polymerase and any deviation from the ideal active site geometry would not allow the conformational change to take place. Indeed, the crystal structures of the closed ternary complexes showed the dNTP situated in a tightly surrounded environment as it stacks between the $3^{\prime}$ base of the primer strand and 
residues of the O helix [14]. The dNTP binds such that it can form a Watson-Crick base pair with the first singlestranded templating base. The dNTP is further coordinated and positioned by interactions to two metal ions located within the active site.

The alignment of the dNTP in the closed conformation allows a two metal ion mechanism for the nucleotidyl transfer to occur. The two metal ions, positioned approximately $3.6 \AA$ apart, are octahedrally coordinated by all three phosphates of the dNTP, the highly conserved side chain residues of the enzyme, and two water molecules (Figure 2). Metal ion A functions to lower the ribose's $3^{\prime} \mathrm{OH}$ affinity for its hydrogen. This makes the attack on the $\alpha$ phosphate of the dNTP by the primer's $3^{\prime} \mathrm{O}^{-}$possible. Metal B serves to support the leaving of the pyrophosphate and together both metals stabilize the negative charge of the pentacovalent transition state. It appears that all polymerases studied to date, as well as enzymes such as HIV -1 reverse transcriptase, utilize this same two metal ion mechanism for nucleotide addition (minireview in [18]).

Similar to the changes within the enzyme during binding, the DNA undergoes several changes from its typical solution based linear B-form duplex structure [8, 13-15]. The DNA in the ternary complex remains held in place by the small conformational change of the thumb. This maintenance of the closed thumb conformation ensures that despite continued dissociation and reassociation of incoming dNTPs during the selection process, the transient binary DNAPol complex remains relatively stable and the propensity for dissociation during these transient events does not increase dramatically. The polymerase-DNA interactions are almost exclusively nonspecific in nature, binding to the nonsequence-specific minor groove while providing no interactions to the sequence specific major groove, with the small exception of those bases located near the active site. Despite the wide breadth of organisms the polymerases were obtained from, the DNA is mostly B-form yet transitions to A-form DNA as it nears the active site $[8,13-15]$. The Bform DNA has an ordered spine of water molecules along the minor groove interacting with the $\mathrm{N}^{3} \mathrm{~s}$ of purines and $\mathrm{O}^{\prime}$ 's of pyrimidines [15]. Both proper A-T and G-C base pairs display the correct $\mathrm{N} 3$ and $\mathrm{O} 2$ along the minor groove required for this interaction, whereas misincorporations will cause a disturbance in these interactions, possibly providing a mechanism by which misincorporations can be detected upstream of the active site. These highly ordered water molecules are intentionally disrupted along the minor groove as it nears the active site, giving rise to A-form DNA and a widening of the minor groove with a decreased helical twist in this region. The DNA is further contorted forming a slight " $\mathrm{S}$ " shape, with the first bend being induced by interactions with the palm domain, and the second from interactions with the closed thumb [14].

As the conformational change from the open to closed ternary complex occurs, another interesting movement takes place regarding the templating base and an aromatic residue at the base of the O helix (Tyr766 in Klenow Fragment, Tyr671 in Klentaq, Tyr530 in T7). In both the open binary and open ternary complexes, the templating base is twisted almost $90^{\circ}$ away from the nucleotide binding site (Figure 2(a)), and in its place the aforementioned Tyr671 of the O helix is stacked on top of the template of the terminal base pair [8]. At first glance, this appears to be illogical since the template base must be base-paired with the incoming nucleotide to direct its proper selection. However, this unique positioning of the templating base in the open conformation is thought to allow an incoming dNTP to "preview" the template prior to reaching the full depth of the binding pocket and has thus been coined the preinsertion site [19]. This preview in the preinsertion site may facilitate selection of a correct dNTP early on in the incorporation process. Upon binding of a correct dNTP to the complex, the conformational closing movement of the $\mathrm{O}$ helix induces a movement of the tyrosine side chain out of its stacking arrangement on the template, allowing the flipped out templating base to rotate back into its typical stacking arrangement with the template (Figure 2(b)) [8]. The exchange of positions of the tyrosine side chain with that of the templating base in the closed conformation allows the templating base to form its typical Watson-Crick hydrogen bonding alignment with the incoming dNTP. Given a correct fit, the subsequent alignment of catalytic residues takes place and phosphodiester bond formation can ensue.

However, selection of a proper nucleotide is no trivial matter considering the onslaught of correctly base pairing ribonucleotides and mismatched deoxyribonucleotides that must be rejected in order to find the correctly matching substrate. Utilizing a FRET-based stopped flow and chemical quench assay, Joyce et al. studied the mechanism by which each of these incorrect substrates are selected against during the process of replication [20]. In this assay, a fluorophore donor was positioned on a mobile portion of the fingers of Klenow fragment and a fluorescence quencher was positioned on the DNA. This allowed monitoring of a change in position of the fingers relative to the DNA and the formation of a correctly paired ternary complex. A concentrationdependent decrease in fluorescence was seen in the presence of the next correct dNTP, indicating that fingers closing was occurring. Mispairing dNTPs as well as mispairing rNTPs showed little fluorescence change, suggesting that they are selected against early on in the process, prior to fingers closing. This implied that a large discrimination against mispairing nucleotides (dNTPs and rNTPs) occurs directly in the open conformation. Addition of a correctly base pairing rNTP caused the conformational change to a closed complex to become significantly hampered, yet still occur to a marginal extent. This reduction in conformational change had previously been identified as a direct clash between the $2^{\prime}-\mathrm{OH}$ of the incoming ribonucleotide and the steric gate side chain Glu710 of Klenow fragment (Glu615 of Klentaq, see Figure 2) [21]. Similarly, Doublié et al. postulated, based on their crystal structure of T7 DNA polymerase, that the corresponding Glu480 residue along with Tyr526 forms a hydrophobic pocket that would preclude the $2^{\prime}-\mathrm{OH}$ of ribonucleotides [14].

Taken together, this indicates that the selection of a correctly pairing substrate occurs first, followed by a selection against ribonucleotides. This specific order for the 


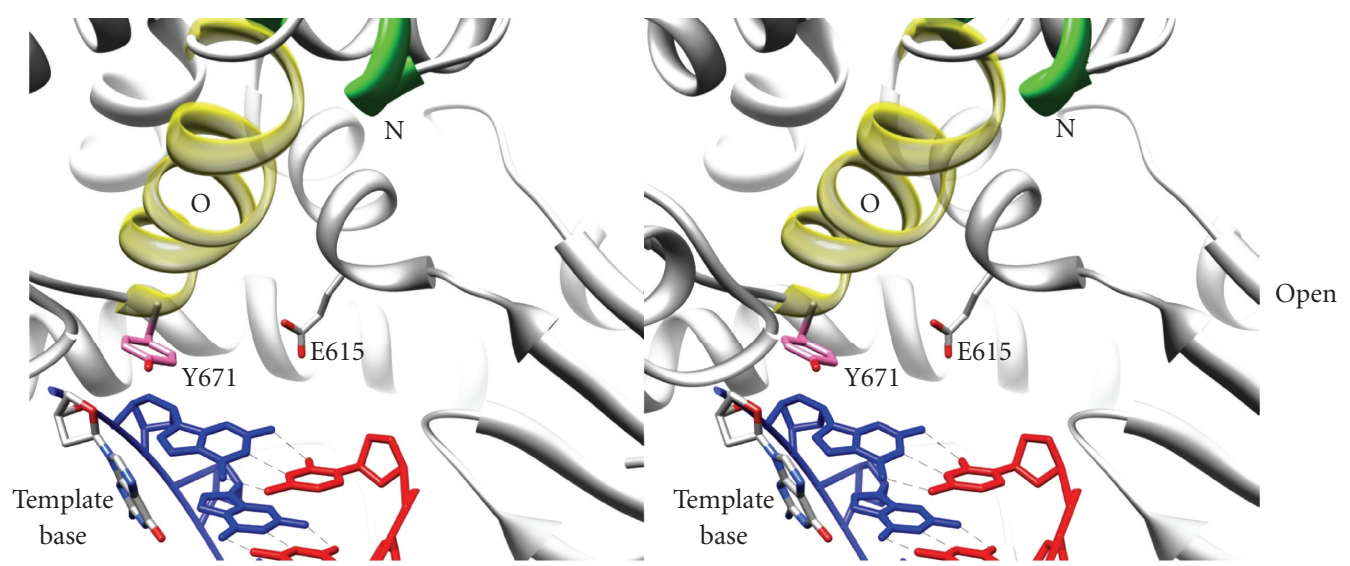

(a)

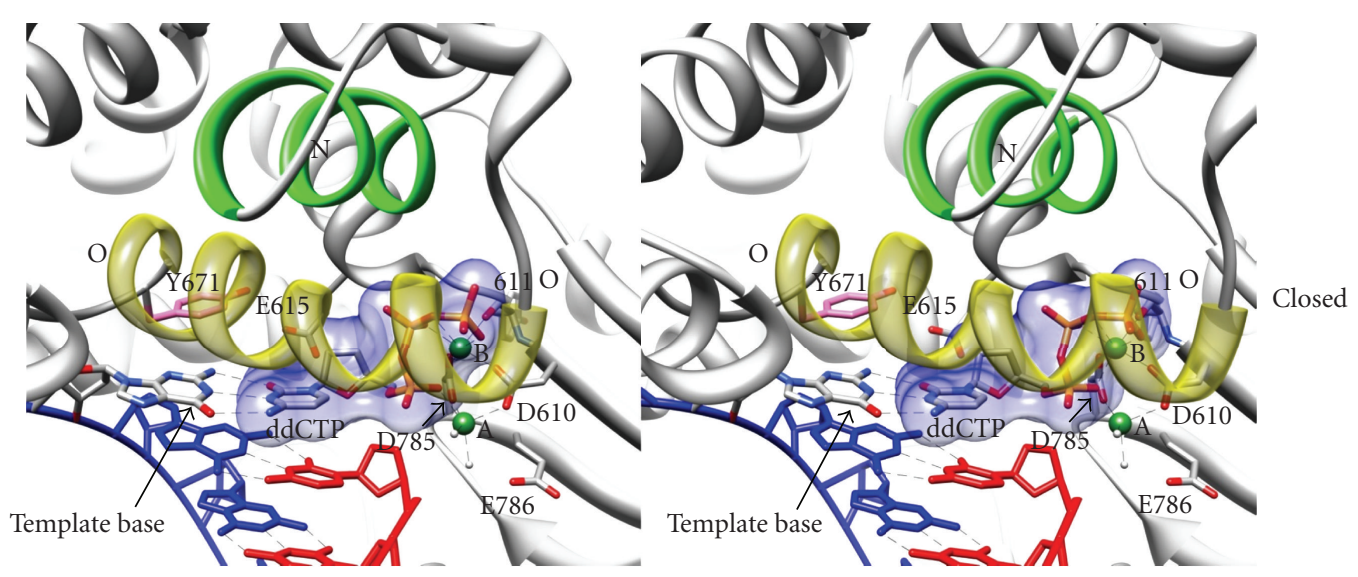

(b)

FIGURE 2: Stereoview (cross-eyed viewing) of Klentaq1 active site in the open binary (a) (PDB \#4KTQ) and closed ternary (b) (PDB \#3KTQ) conformations from the same view point [8]. (a) Open conformation: The O (yellow) an N (green) helices are in an open conformation allowing incoming dNTPs to reach the active site. The templating base (colored by element) is flipped out and Y671 (pink) occupies a stacked arrangement with the template (dark blue). (b) Closed conformation: The $\mathrm{O}$ and $\mathrm{N}$ helices have undergone a large conformational change and closed off the nucleotide binding pocket, which a ddCTP (colored by element with blue space filling) now occupies. The Y671 residue has flipped away from the template allowing the templating base to form a Watson-Crick base-pair with the ddCTP. The position of E615 allows screening of rNTPs via a steric clash with what would be a $2^{\prime} \mathrm{OH}$ of an incoming rNTP. The metal ions A and B (green spheres) form interactions with the phosphates of the ddCTP, and Klentaq residues D610, 611 O, D785, and 2 waters (grey spheres). The missing binding partner of metal $\mathrm{A}$ is hypothesised to be the missing $3^{\prime} \mathrm{OH}$ of the dideoxy-terminated primer terminus.

stepwise selection of a proper substrate provides a more efficient pathway for replication. By initially constraining downstream processing of a substrate to only correctly base pairing molecules, six of the eight possible substrates will be selected against. This provides an efficient first step to screen out as many incompatible substrates as possible, thereby limiting downstream processing of incompatible substrates. In essence, only one incorrect substrate (the correctly pairing ribonucleotide) of the initial eight nucleotide substrates will see further unnecessary processing. Conversely, if the ribonucleotide was selected against first, four of the eight substrates (all dNTPs) would need to be screened for in downstream processing prior to catalysis, and any additional time spent in their processing would be wasted three out of the four times. Overall, the conformationally-induced changes in the thumb upon binding DNA, and the large changes in the fingers, function to grip the DNA and dNTP substrate, respectively. This provides a means for testing the nascent base pair for correct alignment and ensuring that only compatible dNTPs are incorporated.

\section{Roadblocks to Replication}

The mechanism by which a correct nucleotide is chosen is clearly a complex process even under ideal conditions. However, DNA is under constant assault from both endogenous and exogenous agents that alter the DNA template by modifying the bases, resulting in distortions in the DNA structure or changes in the base electrostatics. Due to their disrupting nature, DNA damage can act as roadblocks to DNA polymerases or result in nucleotide misincorporations 
and generation of frameshifts. One class of DNA damage that has been well-studied is that formed when experimental animals are treated with $N$-acetyl-2-aminofluorene (AAF). This compound, originally patented as an insecticide, has been shown to induce the formation of tumors in a variety of organs and was subsequently never introduced to market [22]. Metabolic activation and reaction of these compounds with DNA results in the attachment of the AAF or the related $\mathrm{AF}$ counterpart to the $\mathrm{C} 8$ position of guanine as the major DNA adducts.

Structural, biochemical, and theoretical studies all indicate that the dG-C8-AF structure causes much less distortion in duplex DNA compared with the dG-C8-AAF adduct. NMR experiments show that the guanine bearing the AAF adduct rotates from an anti- to syn conformation (Figure 3 ) in the double stranded DNA helix so that the fluorine moiety becomes inserted into the helix (base displacement model) [23]. This contrasts with the dG-C8-AF adduct that can adopt interchangeable conformations: (i) in the major structure, the fluorene remains outside the helix (outside binding model) and (ii) while the minor conformation has the fluorene ring stacked within the helix [24, 25]. These different conformations presumably are related to the differences observed in the biological properties of these two adducts. Utilizing primer extension assays, numerous studies have shown that a dG-C8-AAF adduct at the templating position poses a strong block to DNA synthesis by highfidelity polymerases, whereas a dG-C8-AF adduct in the same sequence is more easily bypassed [26]. When the adducts are positioned on the template downstream singlestranded region at the +1 and +2 templating positions little to no effect from either adduct is noted. (For clarity, due to the various formats utilized to describe the positions of nucleotides, the numbering scheme used here is such that the first single-stranded templating base found within the insertion site of the polymerase during normal replication is signified position 0 (this is the current base being replicated). Downstream single-stranded template DNA is given consecutive positive numbers beginning with the +1 template base found in the preinsertion site, and upstream duplex DNA already replicated is given consecutive negative numbers starting with the first duplexed base pair in the postinsertion site ( -1 position). This scheme is used to allow a direct visualization of the nucleotide position being discussed in relation to the polymerase, and is also the primary numbering schematic used when discussing crystal structures [27].) In contrast to positioning of the adduct at the +1 and +2 positions, positioning at the postinsertion site (position -1) or in regions of the DNA upstream of the postinsertion site (at the -2 and -3 positions relative to the insertion site) leads to diminished synthesis [28]. This indicates that the specific placement of the adduct within the polymerase active site was guiding its behaviour $[28,29]$.

It is interesting to note that despite the block posed by the presence of the dG-C8-AAF adduct, gel shift binding experiments utilizing Klenow fragment showed that polymerase binding to the AAF modified template was an order of magnitude greater than to native DNA [26]. In addition, unlike native DNA where addition of a correctly base pairing
dNTP induces a tighter binding, the identity and presence of any dNTP had little to no effect on this already strengthened binding. To remedy this counterintuitive discovery, Dzantiev and Romano postulated that the AAF moiety was interacting with hydrophobic amino acid residues located within or near the active site of the polymerase. This interaction was thought to strengthen the binary DNA-polymerase complex and also preclude binding of a dNTP within the active site. Further, the authors postulated that the adduct may block the conformational change seen in the presence of the next correctly base pairing nucleotide, thereby removing any further energetic contribution that the dNTP would bestow upon the complex.

A subsequent paper by the same authors explored more directly the conformational change of Klenow fragment in the presence of these two adducts [29]. Utilizing the same tryptic digestion assay discussed above for unmodified DNA, it was shown that the conformational change typically induced by the presence of the next correct dNTP was in fact inhibited by the presence of the dG-C8-AAF adduct, as predicted. The tryptic digestion band indicative of the open conformation was maintained despite the presence of any dNTP. However, this was only observed when the adduct was in the templating position. When the dG-C8-AAF adduct was moved one nucleotide to the +1 templating position (the preinsertion site), the dNTP-induced conformational change was again observed. Conversely, placement of a dGC8-AF adduct in the templating position (insertion site) only partially inhibited the conformational change to a closed ternary complex. This difference was attributed to the dissimilar conformations that each adduct adopts within the polymerase active site and further strengthened the proposition that the AAF adduct was inducing interactions within the polymerase-DNA complex in a way that precluded the conformational changes necessary for competent nucleotide binding [29].

Picking up on this line of investigation, in 2003 Lone and Romano utilized a Klenow mutant, Y766S, to focus on the specific interactions responsible for the effect of the AAF adducts on this inhibition [30]. In the native protein the tyrosine at position 766 is located at the base of the $\mathrm{O}$ helix near the junction of the fingers and palm domain and is thought to function in maintaining active site geometry. This tyrosine stacks with the templating base in the open conformation and swings away during formation of the closed complex (similar to Figure 2(b)), (see more detailed description in Section 3). Mutations at this position have been shown to both increase the rate of insertions of incorrect nucleotides and reduce the ability to extend from these misincorporations [31]. Under identical conditions, the wild-type Klenow fragment predominantly stalled one nucleotide prior to the dG-C8-AAF adduct, incorporating across from the adduct approximately $20 \%$, and extending past the AAF adduct to yield $6 \%$ full extension. Interestingly, the Y766S mutant showed a higher (40\%) incorporation across from the AAF adduct, but gave only $1 \%$ full extension [30]. In addition, the Y766S mutant showed a 16-fold higher $V_{\max } / \mathrm{K}_{m}$ for incorporation of a correct $\mathrm{dC}$ across from the dG-C8-AAF adduct and also displayed a much greater 


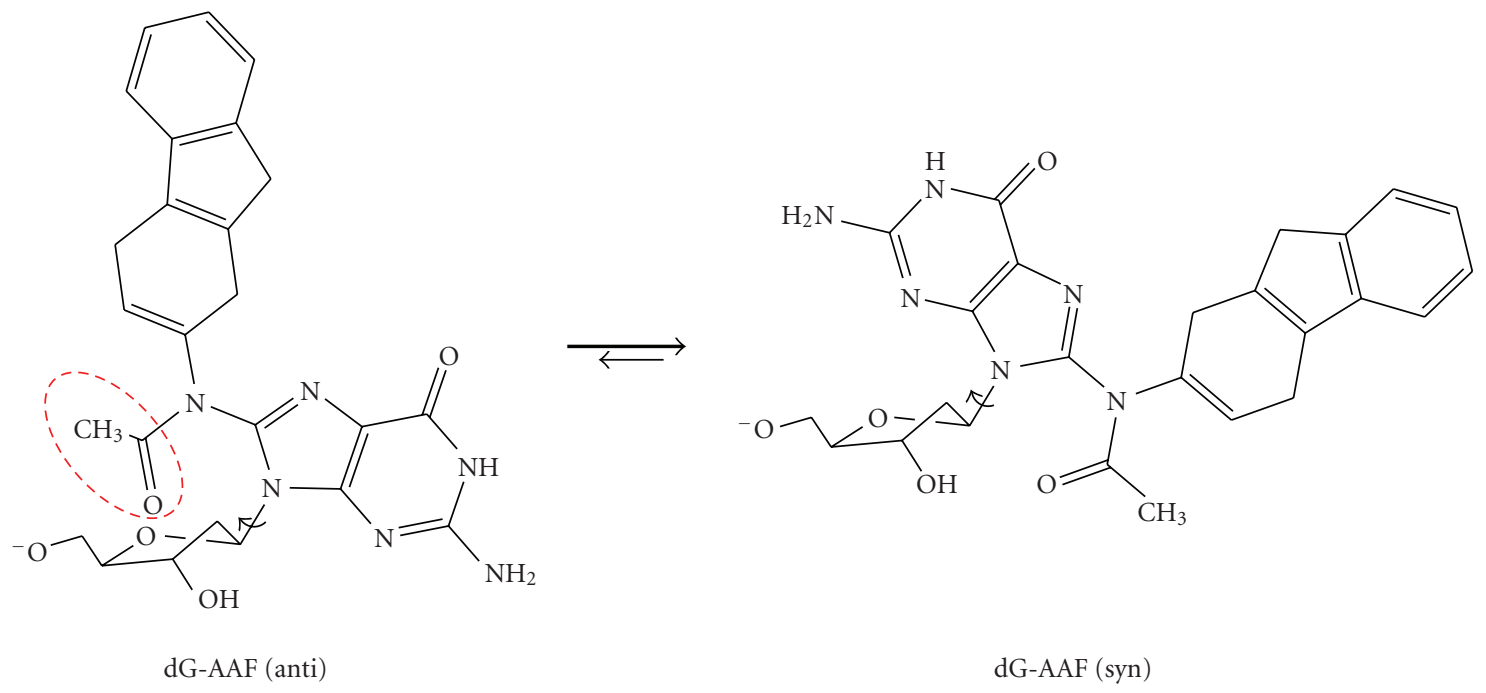

FIgure 3: Conformations of a dG-C8-AAF adduct. Native DNA favors the anti conformation that allows Watson-Crick base pairs to form with the opposing strand while a dG-C8-AAF in the anti conformation results in a steric clash between the acetyl moiety (circled red) and the ribose. This causes the conformational equilibrium to shift towards the more favorable syn conformation. dG-C8-AF adducts differ from dG-C8-AAF adducts by having a smaller hydrogen in place of the circled acetyl moiety. This avoids the steric clash and allows both the synand anti conformations to be sampled.

propensity to misincorporate nucleotides on unmodified DNA. Together, this illustrated the importance placed upon the tyrosine residue for discrimination of a correct base pair and also shows that the Y766S mutation allowed the accommodation of the AAF-C8-dG lesion, possibly because of a more open active site that allows improper base pairs to form during synthesis [30].

Using gel shift analyses and the tryptic digestion assay, the ability of the Y766S mutant to undergo a conformational change was examined [30]. Similar to wild-type, the Y766S mutant showed an increased binding to native DNA in the presence of the next correct dNTP. The binding of the mutant polymerase to a dG-C8-AAF adduct was also increased, again similar to wild-type. However, where the wild-type showed no further increase in binding strength to dG-C8-AAF containing primer-templates in the presence of a correctly pairing dCTP, the mutant polymerase did show increased binding. In fact, the presence of any nucleotide appeared to increase the binding strength of the mutant, indicating a possible conformational change was occurring. This was confirmed utilizing the tryptic digestion analysis in which it was shown that the presence of dCTP caused protection of the cleavage site whereas other dNTPs reduced cleavage, indicating a conformational change was taking place despite the presence of the dG-C8-AAF adduct [30]. The ability of the mutant $\mathrm{Y} 766 \mathrm{~S}$ to undergo a conformational change even in the presence of a bulky dG-C8-AAF adduct is indicative of the more open active site and the importance the tyrosine residue plays in this conformational transition. Interestingly, the $\mathrm{Y}$ family of bypass polymerases that specialize in synthesis past bulky adducts also display a markedly open active site [33]. It is thought that this open nature better accommodates lesions by reducing the propensity of steric clashes, although at the expense of reduced fidelity.

\section{What You See Is What You Get}

It has long been thought that the conformations adopted within the active site by various DNA adducts will account for the array of biological effects and mutagenic consequences that the adducts display [22,34]. Direct evidence for the predicted mechanism of inhibition of the conformational change for wild-type Klenow fragment in the presence of dGC8-AAF, along with the increased binding affinity to these substrates, came in the form a crystal structure published in 2004 [32]. Dutta et al. were successful in obtaining functionally relevant crystal structures of bacteriophage T7 DNA polymerase bound to DNA duplexes containing either a dG-C8-AAF or dG-C8-AF at the templating position. The crystal structure of the dG-C8-AAF adduct has several key features that correlate with the aforementioned results obtained by Romano et al. First, the polymerase complex with dG-C8-AAF modified DNA is in a distorted open conformation and no nucleotide is bound to the complex despite the fact that crystals were growth in the presence of ddCTP (Figure 4(a)). The modified nucleoside is in a syn conformation, flipped out of the active site and bound to the surface of the fingers. The AAF moiety is positioned in a hydrophobic pocket behind the $\mathrm{O}$ helix stacking alongside Phe528, which is usually buried within the fingers (Figure 4(b)). Further stabilizing the position of the AAF moiety are hydrogen bonds between the adducted guanine's N2 and N7 with Asp534 and Arg566, respectively. The AAF interactions with the $\mathrm{O}$ helix pushes the helix towards the active site, forcing Tyr530 (analogous Tyr766 of Klenow fragment and Tyr671 of Klentaq) partially into the nucleotide binding site [32]. The positioning of the AAF and its various direct interactions with the polymerase, as well as the inability to bind nucleotide due to the positioning 


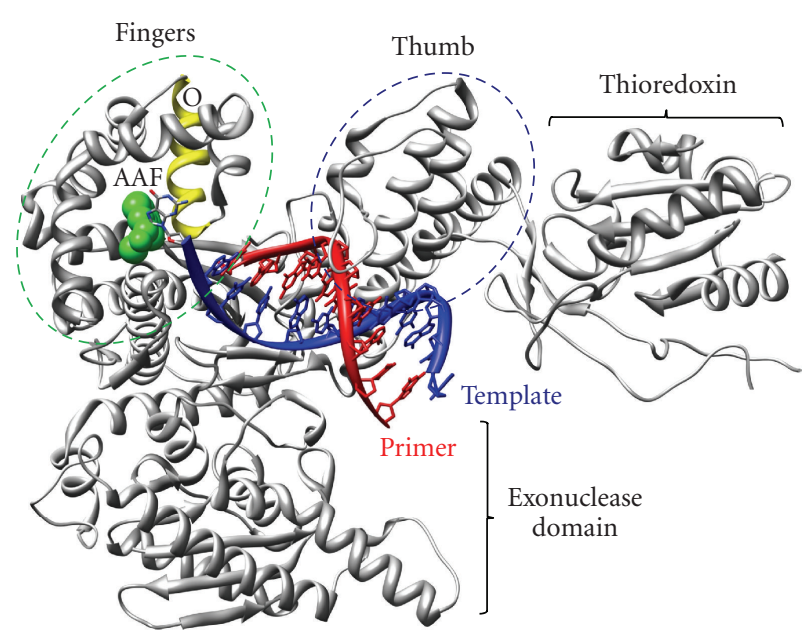

(a)

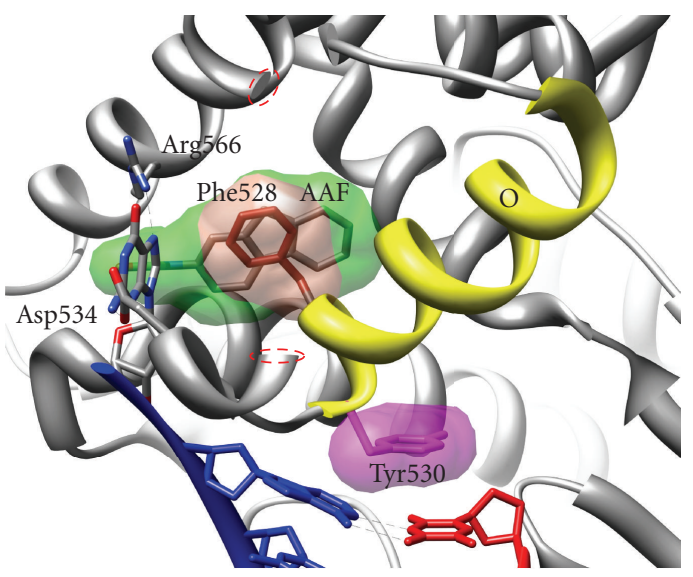

(b)

FIGURE 4: Structure of bacteriophage T7 polymerase bound to a DNA primer-template containing a dG-AAF adduct at the templating position (PDB\# 1X9M) [32]. (a) The overall shape of T7 polymerase resembles a human right hand forming fingers (green circle), thumb (blue circle), and palm (not highlighted). T7 also contains thioredoxin, a processivity factory, in addition to the polymerase and exonuclease domains. The AAF adduct (green) inserts behind the O helix (yellow), locking the helix in a distorted open conformation, inhibiting the binding of dNTPs. (b) Close-up view of active site. The AAF moiety (grey with green space filling) is inserted into a hydrophobic pocket behind the $\mathrm{O}$ helix stacking with a flipped out Phe528 (brown, with brown space filling), which is buried in the native structure. The adducted guanine is in an anti conformation placing the hydrogen bond acceptors and donors away from the nucleotide binding position. This position is further stabilized by hydrogen bonds to Arg566 and Asp534. The AAF moiety pushes the O helix forward, causing Tyr530 (pink with pink space filling) to stack on top of the DNA, occupying the nucleotide binding site which occludes a dNTP from binding. Residues 537-557 have been removed for simplified visualization (ends are circled red).

of Tyr530, explain why the adduct places a strong block on replication as well as its increased binding affinity. This also confirms the previous experiments performed using Y766S that showed that the presence of the smaller serine allowed the conformational change to a closed ternary complex to occur.

The crystal structure containing the analogously positioned dG-C8-AF adduct showed similar conformations as the AAF adduct, but with a few significant differences. The AF residue was not positioned in the same hydrophobic pocket as its AAF counterpart, but instead the electron density of this moiety was either of poor quality or absent [32]. Despite limited quality of the electron density around the AF adduct, it could be established that the AF moiety was also not binding to the active site of the polymerase. This is indicative of a fluctuation between various conformations, possibly a transition from anti- to syn conformations. The amino acid residues forming the hydrophobic pocket of the AAF complex were oriented in more native-like positions within the AF containing structure. Also, the important Tyr530 was found to occupy the binding site of the template base in the closed ternary complex. The authors modeled the positions of the AF adduct in both syn- and anti- conformations of the nucleoside [32]. The anti conformation was shown to be able to form a closed complex with no significant steric clashes with the AF adduct. However, the syn conformation was shown to place the AF adduct stacked in the active site. This stacking arrangement will effectively compete with the stacking arrangement of guanine in the alternative anti conformation. The positioning of the adducted guanine in the syn conformation would not allow base pairing with an incoming nucleotide and would subsequently stall synthesis when in this conformation.

A paper published by $\mathrm{Hsu}$ et al. in the same year was successful in crystallizing thermophilic Bacillus DNA polymerase I fragment in the presence of an $\mathrm{AF}$ and $\mathrm{AAF}$ adduct, and provided direct visualization of the effects of a dG-C8-AF adduct [35]. Bacillus DNA polymerase I fragment has the ability to catalyze nucleotide addition within the crystal, allowing snapshots to be taken of the adducted guanine within the pre- and postinsertion sites [13]. Capture of a dG-C8-AF adduct within the preinsertion site showed the adducted template base within the preinsertion site in a syn conformation, with the fluorene moiety also buried within the confines of the same site. The presence of the adduct induced a perturbation of the $\mathrm{O} 1$ helix, yet the $\mathrm{O}$ helix maintained its position in an open conformation. The remainder of the protein and the DNA was unaltered by the presence of the dG-C8-AF adduct. This showed that prior to incorporation of a dCTP the dG-C8-AF was positioned within the preinsertion site, whereas the catalytic site, the $\mathrm{O}$ helix, and postinsertion site remained unaffected and presumably capable of performing the transfer of the template base from the preinsertion site to the insertion site. The relatively ordered structure allows nucleotide incorporation to occur opposite the dG-C8-AF adduct, albeit to a reduced extent relative to unmodified DNA. 
After a dCTP had been incorporated within the complex, the dG-C8-AF adopts an anti conformation, placing the AF moiety within the major groove while the $\mathrm{dG}$ forms cognate Watson-Crick hydrogen bonds with the $\mathrm{dC}$ within the postinsertion site. The polymerase is able to accommodate this structure because the adduct is solvent exposed within the major groove, unlike what would occur if its placement was into the minor groove where many interactions between the DNA and the polymerase are found. This conformation is similar to the outside model that places the AAF moiety outside the duplex DNA helix [24, 25]. This structure also showed a much greater degree of distortion that ultimately affects DNA replication. The dG$\mathrm{C} 8-\mathrm{AF}: \mathrm{dC}$ induces distortions to the template that relocate the $n-1$ template base causing perturbations to the minor groove, the $\mathrm{O}$ and $\mathrm{O} 1$ helicies, and placing the AF moiety obscuring the preinsertion site to the next template base. This conveniently explains the ability of polymerases to incorporate across from an $\mathrm{AF}$ adduct, yet exhibit difficulty in extending further. Conversely, the dG-C8-AAF adductcontaining structure in the pre- and postinsertion sites were indistinguishable, and hence a dCTP was absent from the postinsertion structure. In both structures the protein was in an open conformation with an empty preinsertion site as the AAF-adducted guanine is somewhat disordered and placed over the preinsertion site. The duplex DNA, as well as the protein's catalytic, pre- and postinsertion sites were undisturbed. Again, this inability to perform incorporation could presumably be due to the inability of a dG-C8$\mathrm{AAF}$ adduct to adopt an anti conformation. In general, the observed crystal structures Dutta et al. [32] and of Hsu et al. [35] correlated well with the previous results and provided further support for the premise that the effects of these adducts are related to the conformations that they adopt within the polymerase active site. However, the crystal structures of Dutta et al. and Hsu et al. show that the conformations adopted by the adducts are also dependent upon the polymerase they are bound to, yet still correlate well with the previously observed results.

\section{Not All Sequences Are Created Equal}

The stark difference in the mutagenic properties of AAF and $\mathrm{AF}$ adducts are further influenced by the sequence of bases surrounding the adduct. AAF's ability to induce frameshifts is targeted to repetitive sequences such as the NarI restriction recognition sequence $\left(5^{\prime}-\mathrm{G}_{1} \mathrm{G}_{2} \mathrm{CG}_{3} \mathrm{CC}-3^{\prime}\right)$ (NarI sequence reviewed in [36]) [37]. In bacteria, when an AAF adduct is positioned at $\mathrm{G}_{3}$ a dinucleotide $\mathrm{GC}$ deletion is produced, while an AF adduct at this same position does not yield the deletion product $[37,38]$. The dinucleotide deletion is thought to be induced via a slipped displaced structure where during synthesis the primer is capable of misaligning with the template within the polymerase active site. These misaligned looped out structures are further stabilized by Watson-Crick base pairing of adjacent nucleotides, allowing the polymerase to skip the nucleotides in proximity to the adduct. Gill and Romano explored this possibility utilizing primer extension analysis and gel shift binding assays of various primers along the NarI restriction recognition sequence [39]. By positioning various primers along the AAF modified template and examining which nucleotide best complemented binding of Klenow fragment, along with the ability to extend the substrate, it was shown that the formation of a GC dinucleotide bulge was induced. Interestingly, where the AAF adduct previously had caused a significant increase in binding to non-NarI sequences, an AAF adduct positioned at $G_{3}$ of the NarI sequence showed no increase in binding affinity over the unmodified NarI sequence. In fact, both the binding of Klenow fragment to AAF modified and unmodified NarI sequences showed decreases over non-NarI sequences [39]. This indicated that a possible different conformation with the AAF modified NarI sequence existed within the polymerase active site than had previously been noted in the non-NarI sequence of the T7 or Bacillus fragment crystal structures. Similar to non-NarI sequences modified with AAF, the addition of nucleotides did not enhance Klenow fragment binding. However, the presence of dGTP, dATP, or dTTP did decrease the binding affinity. This was indicative of a destabilizing effect these nucleotides had on the complex, presumably due to an incorrect base pair match.

Taken together, these results indicated a specific twostep mechanism for formation of the dinucleotide deletion (Figure 5). The first step has a dCTP be incorporated across from the AAF modified base, followed by a structural rearrangement that places the AAF modified base along with the upstream $3^{\prime} \mathrm{C}$ in a dinucleotide bulge. The primer's $5^{\prime}$ upstream guanine and the cytosine initially incorporated across from the adduct now base pair downstream of the templates adducted guanine. This two-step mechanism also suggests that the frameshift extension product could be generated in vivo by two different polymerases, one better suited for incorporation across from the bulky AAF adduct followed by another more suited for extension from the adduct (reviewed in [40]). This two enzyme stepwise mechanism has been postulated for both bacterial and eukaryotic polymerases and provides a mechanism to reconcile the different properties required of a polymerase for replicative DNA synthesis, and the specialized niche of performing replication in proximity to bulky DNA adducts. This process is exacerbated by specific sequences such as NarI that preferentially adopt structures to induce frameshift mutations after incorporation of a nucleotide opposite the adducted base.

\section{Bypassing Roadblocks}

A class of polymerases capable of performing nucleotide incorporation in the presence of such distorting adducts as $\mathrm{AF}$ and AAF are the bypass or translesion synthesis (TLS) polymerases [42]. TLS polymerases are characterized by their open active sites that are capable of accommodating bulky lesions and the DNA distortions they produce. For example, yeast $\mathrm{Pol} \eta(\gamma \mathrm{Pol} \eta)$ has a more open active site 


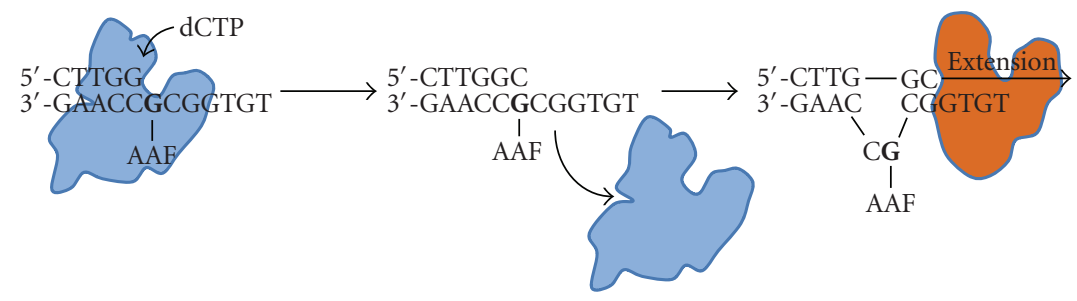

FIGURE 5: Mechanism for bypass of a dG-C8-AAF adduct in the NarI sequence. A polymerase more suited for incorporation across from the adduct places a proper dCTP:G base pair at the adducted position followed by incorporation, then dissociation of the enzyme. A subsequent rearrangement of the DNA to form a 2 nucleotide budge ensues, allowing a possible second polymerase to perform extension from this slipped structure, resulting in a -2 deletion frameshift product.

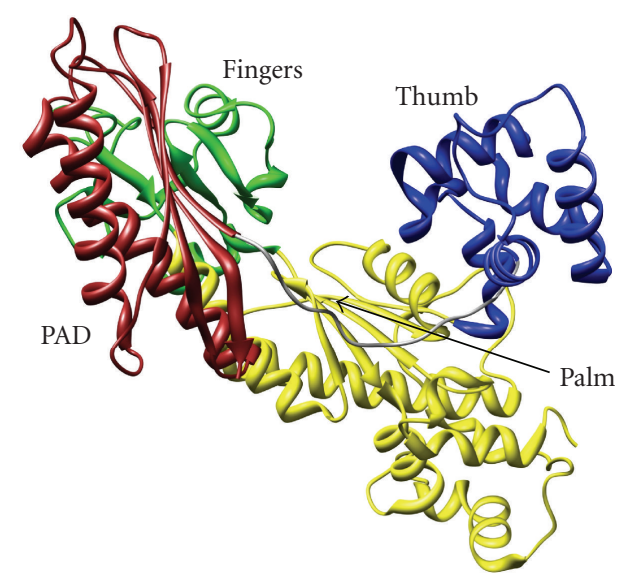

FIgURE 6: Structural overview of the TLS yeast polymerase eta (PDB\# 1JIH, molecule A) [41]. $\gamma$ Pol $\eta$ contains stubby fingers (green), and thumb (blue) domains, a palm (yellow) domain, as well as a polymerase associated domain (PAD) (brown). The open nature of the active site allows two nucleotides to be accommodated during synthesis, as well as bulky carcinogenic adducts and DNA distorting lesions.

caused by stubby fingers and thumb domains, as well as an additional polymerase associated domain (PAD) (Figure 6). $\gamma$ Pol $\eta$ belongs to the Y-family of polymerases and also contains a little finger domain that is unique to this class of polymerases. The more open active site allows Pol $\eta$ in both humans and yeast to correctly synthesis past a cissyn thymine-thymine UV-induced dimer [43]. Unlike the solvent-excluded tight constraints of replicative polymerases, the active sites of Y-family polymerases are solvent exposed and thus capable of accommodating many distorting lesions. However, the open active site comes at the cost of low fidelity, orders of magnitude lower than replicative polymerases [44, 45]. Bypass polymerases typically also do not possess high processivity, indicative of their brief role in synthesis past a lesion then allowing a more precise replicative polymerase to continue synthesis [46].

Prakash and co-workers were the first to present evidence that $\gamma \mathrm{Pol} \eta$ was capable of undergoing a conformational change step prior to chemistry occurring [41]. Utilizing presteady state kinetics and measuring the elemental effect of a sulfur substituted for an oxygen at the $\alpha$-phosphate of the nucleotide they showed that $\gamma \operatorname{Pol} \eta$ undergoes an induced fit mechanism for nucleotide incorporation. $\gamma \mathrm{Pol} \eta$ poorly discriminates between correct and incorrect nucleotides both at the initial nucleotide binding step and during the induced conformational change. A more direct FRET-based method was used to visualize the global conformational dynamics of another similar Y family polymerase, Dpo4, in a paper published by $\mathrm{Xu}$ and co-workers [47]. Here, FRET pairs were positioned at various locations around Dpo4 allowing movement of specific locations to be tracked during real time measurements. Assemblage of all movements along with the rates of the corresponding FRET changes allowed the authors to assemble global movements of the enzyme. Upon binding of a correct nucleotide, a concerted global rearrangement of all four domains (fingers, little fingers, thumb, and palm) takes place where the little fingers domain moved in opposing directions to the polymerase core domains. The movement was relatively small compared to those of T7, being only a few angstroms, yet form similar fingers and thumb closing motions as mentioned previously for various other polymerases.

On unmodified DNA, gel shift binding assays with $\gamma \mathrm{Pol} \eta$ showed increased binding in the presence of the next correct nucleotide [48]. This is consistent with a dNTP-induced conformational change resulting in a closed ternary complex, just as what had been shown previously for Klenow fragment. Addition of an incorrectly base pairing dGTP was noted to have a destabilizing effect on the complex, presumably due to an incompatible fit within the enzyme, again similar to that previously observed for Klenow fragment.

Extension assays showed that $\gamma \mathrm{Pol} \eta$ is capable of fully bypassing a dG-C8-AF adduct and while incorporation occurred across from a dG-C8-AAF adduct, extension past this position was inhibited [48]. These results are similar to that observed for the Klenow mutant Y766S, which, similar to $\gamma \operatorname{Pol} \eta$, also has a more open active site. Interestingly, where Klenow fragment was unable to become more stable upon addition of any dNTP across from a dG-C8-AAF adduct, binding was stabilized with $\gamma \operatorname{Pol} \eta$ in the presence of $\mathrm{dCTP}$, consistent with the formation of a closed ternary complex. Also, consistent with the lack of extension after incorporation across from the AAF adduct, no enhanced binding was observed at this position in the presence of the next correct nucleotide. Despite the structural 


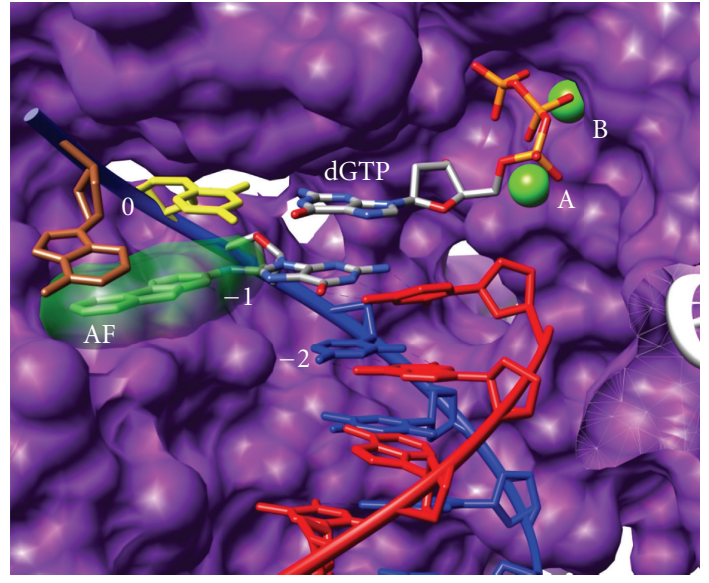

(a)

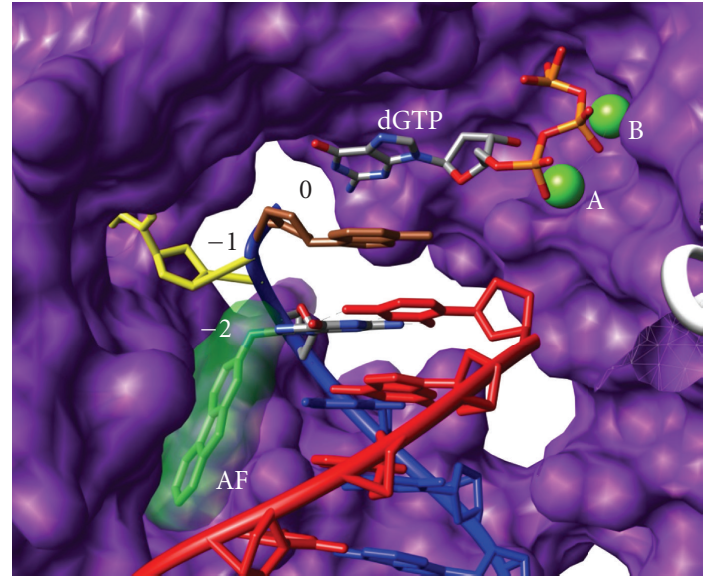

(b)

Figure 7: Two structures of Dpo4 ternary complexes in the presence of a dG-C8-AF adducted template, one forming a correctly aligned primer-template and a second forming a looped out structure of the template (PDB\# 3KHH) [27]. (a) Correctly aligned primer template with the $\mathrm{dGTP}$ and corresponding template $\mathrm{dC}$ correctly at the 0 position. The adducted $\mathrm{dG}$ retains base paring with its corresponding dC while the AF is positioned towards the major groove and stacks in a pocket of the little fingers domain. The +1 templating base stacks on top of the AF adduct and is in alignment with the 0 position. (b) The misaligned structure contains the templating base in a looped out structure. The looped out base slides into a pocket, rotated away from the active site. As a consequence the +1 templating base rotates and stacks on top of the adducted dG base, and the DNA is shifted downwards as the +1 base now occupies the -1 position within the polymerase. The AF adduct is rotated downwards and placed into a hydrophobic pocket within the little fingers domain. The dGTP does not contain any Watson-Crick binding partner as it occupies the 0 position and stacks onto of the template base below.

and behavioral differences between replicative polymerases and lesion bypass polymerases, a common conformational change in the mechanism appears to be shared in this case. It should be noted, however, that studies of some polymerase such as Dbh have not been able to detect a conformational change [49]. Although it is conceivable that smaller undetected conformational changes are performing the analogous function as the larger scale rearrangements seen in replicative polymerases or that these motions have yet to be detected.

The open active site of Y-family polymerases is not necessary only to accommodate DNA lesions, but also able to allow various primer-template alignments that may favor the replication of the adducted template. As mentioned previously, the formation of frameshift mutations in the NarI sequence occur via a template misalignment and this misaligned structure must be accommodated within the polymerase active site. A recent crystal structure by Rechkoblit et al. of the $\mathrm{Y}$ family polymerase Dpo4 in the presence of an dG-C8-AF adduct shows such a complex and how it can be accommodated and stabilized within the polymerase [27].

In this study, two molecular structures were obtained in one asymmetric unit of the crystal: (1) a correct alignment with the dG-C8-AF adduct base pairing with a dC at the -1 position (dG-AF: $\mathrm{dC}$ at the postinsertion site) and a typical correctly forming dC:dGTP pair of the adjacent base ( $5^{\prime}$ template side of adduct at position 0 , the insertion site) (Figure 7(a)) and (2) a misaligned structure with the dG$\mathrm{C} 8$-AF adduct base pairing with a $\mathrm{dC}$ shifted to the -2 position (1 position upstream of the postinsertion site) and the adjacent $5^{\prime} \mathrm{C}$ of the template looping out (Figure 7(b)). More specifically, in the correctly aligned molecule 1 the dG containing the $\mathrm{AF}$ is in an anti conformation and the $\mathrm{AF}$ is placed on the major groove side of the duplex within a pocket buried from solvent, and stabilized by interactions to the little finger domain. This is similar to the conformation of the AF adduct in Bacillus fragment, with the exception that Dpo4 contains interactions to the little fingers domain that stabilize the adducts position, a caveat that is impossible in Bacillus fragment due to the lack of a little fingers domain [35]. The template $\mathrm{C} 5^{\prime}$ of the adduct (at position 0 , the insertion site) is base paired with an incoming dGTP, yet is shifted from its typical stacking arrangement over the postinsertion site base pair, and is instead poised partially over the AF adduct with the dGTP over the center of the postinsertion site base pair. In addition, the next downstream template base ( 0 position, which would typically be within the insertion site) is flipped out and stacks on top of the AF adduct in alignment with the templating $\mathrm{C}$ base and the incoming dGTP at the 0 position (insertion site). In the misaligned structure of molecule 2 the dG-C8-AF: $\mathrm{dC}$ base pair is shifted upstream relative to the polymerase to the -2 position, as the adjacent $5^{\prime}$ template $\mathrm{C}$ loops out of the helix towards the major groove, stacking with the little fingers domain (Figure 7(b)). Again, the $\mathrm{AF}$ adduct is on the side of the major groove and situated within a pocket on the little fingers. The 0 template base is rotated back into the helix and occupies the postinsertion site $(-1$ position) as the incoming dGTP stacks on top of it in the insertion site (occupying the 0 position). Consequently, the incoming nucleotide does not possess a Watson-Crick hydrogen binding partner. 
Interestingly, the correctly pairing ternary structure of molecule 1 was poised in resemblance to a binary like state which is incapable of performing catalysis without additional rearrangements. However, the misaligned structure of molecule 2 showed an uncanny resemblance to the ternary complex of Dpo4, with protein-DNA interactions similar to that of the ternary complex. It should be noted however that the misaligned structure of molecule 2, despite being arranged similar to a ternary complex, would also require further rearrangements in order to achieve a catalytic state.

The ability of Dpo4 to not only accommodate the bulky AF lesion but also to stabilize its position by binding to the little fingers domain that only $\mathrm{Y}$ family polymerases possess, is indicative of its design for its role in lesion bypass. In addition, Dpo4's ability to further utilize its specialized little finger domain to stabilize primer-template misalignments strengthens its ability to cope with distorting lesions, albeit at the expense of increasing mutagenicity. This paper also shows that misaligned structures are not only stabilized by the Watson-Crick base pairing of the misaligned structure, but also, and possibly more so by direct interactions of the polymerase with the misaligned complex [27].

\section{Always on the Move}

Recently, a new picture regarding the conformational dynamics of polymerases has begun to emerge. Singlemolecule assays allow the direct monitoring of individual molecules and can reveal properties that are otherwise masked by ensemble averaging of large populations of molecules. Nonsynchronous fluctuations that appear in fleeting moments of time go unseen in ensemble averages yet become obvious when viewed at the single molecule level. Single-molecule FRET (smFRET) experiments where a FRET donor was positioned on the template of a duplex DNA strand, and a FRET acceptor was positioned on Klenow fragment, allowed the direct visualization of DNA synthesis as it proceeded through incorporation of three nucleotides [50]. The movement of the DNA to the exonuclease domain, as well as the movements during synthesis, could also be viewed. As expected, discrete steps in the FRET traces were observed for each nucleotide incorporation event. Further, a few individual molecules were noted to have the primer transition directly from the polymerization site to the exonuclease site, and vice versa, without dissociation of the binary complex. This correlated well with the proposition made during the modeling of the crystal structure in 1993 by Beese et al. [11]. In addition, following each incorporation event the polymerase appeared to move one nucleotide further downstream than was necessary to place the following nucleotide in the preinsertion site. This was immediately followed by a return back to the expected FRET level for the ensuing preinsertion site. This transient drop was routinely observed in the presence of the next correct nucleotide and was never observed in the presence of an incorrectly base pairing nucleotide. The ubiquitous nature of this novel step led the authors to conclude that this was indeed an integral step in nucleotide incorporation and that it might be involved in checking for proper base pair formation. Movement to this site might be part of the routine proofreading process that results in movement of a misincorporation to the exonuclease domain. This transitioning process to the exonuclease domain was observed when experiments were performed using mismatched primertemplate termini [50]. Alternatively, it was also suggested that the transient state could represent an equilibrium between the preinsertion and insertion complexes or it could represent a further conformational rearrangement that is part of the polymerase mechanism. Regardless, the smFRET based discovery of this novel motion of the polymerase shows new dynamics previously unobserved in bulk biochemical experiments.

Further demonstration of the lively dynamic nature of these enzymes comes from new evidence regarding the continual conformational changes that polymerases undergo during nucleotide incorporation and the identification of yet another conformational state [51]. Santoso et al. utilized alternating-laser excitation (ALEX) smFRET with both members of the FRET pair placed on Klenow fragment to directly monitor conformational changes of the polymerase in real-time. More specifically, one fluorophore was positioned on residue 744 of the fingers domain of Klenow fragment, and the other on residue 550 of the thumb. Using a femtoliter observation volume where individual molecules diffuse freely, the conformational changes of the enzyme could be accurately tracked. Observations of Klenow fragment bound to DNA in a binary complex revealed that despite the lack of dNTP, the enzyme was found to occupy both the open and closed states, with the open conformation existing $66 \%$ of the time. The large occupancy of polymerases in a closed binary complex is a fascinating proposition and adds an active quality to the crystal structure snapshot of the binary complex published almost two decades previously [11]. Upon addition of the next correctly base pairing dNTP, the closed conformation dominated $84 \%$ of the time [51]. Addition of incorrectly base pairing dNTPs revealed a third conformation of the enzyme that was distinct from the open and closed complexes identified previously. Similarly, addition of rNTPs also showed the formation of a third state. Despite the similarities observed in the third conformational state observed with incorrect dNTPs and rNTPs the authors advocate that they cannot be the exact same state, because as noted earlier the rejection of an incorrectly base pairing dNTP occurs at a different step along the reaction pathway than does the rejection of rNTPs. Regardless, the third state is thought to more closely resemble an open KF-dNTP complex where the interactions of the triphosphate moiety induce a subtle rearrangement of the residues along the $\mathrm{O}$ helix, yielding the third FRET state. This process would allow the complementarity of the templating base with the incoming dNTP to be tested in the open conformation. Provided a good fit is obtained the polymerase would then move the pair to the active site via rearrangements of the O-helix as the closed conformation is formed, whereas mispairing nucleotides would be rejected in the open conformation. More striking than the identification 
of the novel third conformational state was the observations that the unbound enzyme was found to be continually fluctuating between various conformational states despite the lack of a DNA template or dNTPs [51]. These conformational transitions were incredibly rapid, occurring in the low millisecond range. The ability of the polymerase to continually sample its conformational states in the absence of substrate further exemplifies the dynamic nature of this enzyme.

\section{Conclusions}

DNA polymerases are remarkable enzymes that are able to match a dNTP with the template base, reject the rNTP that is present at a much higher concentration, accommodate four different base pair orientations in their active sites and do all of this at an incredible rate. The overall threedimensional structure of various polymerases, mainly their anthropomorphic projection of a human right hand, has proven to be strikingly similar from different organisms. Along with these structural similarities, the identification of several key conformational changes appears to be common. These include the subtle rearrangements of the thumb domain of the polymerase to strengthen the grasp on the DNA upon formation of a binary complex and the large nucleotide-induced conformational change of the $\mathrm{O}$ and $\mathrm{N}$ helices upon formation of a ternary complex. The conformational change of the $\mathrm{O}$ helix has been associated with various steps of the mechanism: the alignment of the catalytic residues and the positioning of the DNA; the positioning of indexing residues such as Tyr766 of Klenow fragment; the positioning of the dNTP substrate to lock it in the proper orientation for catalysis; and obstructing incompatible residues such as rNTPs from achieving catalytic competency. DNA adducts have been shown to inhibit DNA synthesis and the ability of adducts such as AF and AAF, to induce radically different effects during synthesis has been linked to the structures they adopt within the active site of polymerase and their ability to disrupt or block conformational changes. The sequence surrounding an adduct further contributes to the mutagenic properties, as is seen with the GGCGCC NarI sequence containing an AAF adduct. Lastly, the view of these enzymes exhibiting stopped motion as they progress through discreetly identified steps is changing. Single-molecule studies have revealed properties masked before in ensemble averaging. Identification of obligate novel intermediates along the mechanistic pathway, coupled with the dynamic nature of these enzymes to sample multiple states, even in the absence of substrates, are two new discoveries that open the door for future studies. It is hoped that future experiments examining these novel changes in the presence of bulky carcinogenic adducts will identify additional conformational checkpoints, and in keeping with anthropomorphic qualities, areas of indecision by polymerases which contribute to mutagenesis.

\section{Abbreviations}

$\begin{array}{ll}\text { dNTP }: & \text { Deoxyribonucleoside } 5^{\prime} \text {-triphosphate } \\ \text { DNA: } & \text { Deoxyribonucleic acid } \\ \text { rNTP: } & \text { Ribonucleoside } 5^{\prime} \text {-triphosphate } \\ \text { ddNTP: } & \text { Dideoxyribonucleoside } 5^{\prime} \text {-triphosphate } \\ \text { AF: } & \text { 2-aminofluorine } \\ \text { AAF: } & N \text {-acetyl-2-aminofluorene } \\ \text { dG-C8-AF: } & N \text { - }\left(2^{\prime} \text {-deoxyguanosin-8-yl)-2- }\right. \\ & \text { aminofluorine } \\ \text { dG-C8-AAF: } & N \text { - }\left(2^{\prime} \text {-deoxyguanosin-8-yl)- }\right. \\ & N \text {-acetyl-2-aminofluorene } \\ \text { TLS: } & \text { Trans lesion synthesis } \\ \text { RNA: } & \text { Ribonucleic acid } \\ \text { FRET: } & \text { Förster resonance energy transfer } \\ \text { smFRET: } & \text { Single molecule Förster resonance energy } \\ & \text { transfer } \\ \text { ALEX: } & \text { Alternating laser excitation. }\end{array}$

\section{Acknowledgment}

This paper was supported by Public Health Service Grant CA40605 awarded by the Department of Health and Human Services.

\section{References}

[1] T. A. Kunkel and K. Bebenek, "DNA replication fidelity," Annual Review of Biochemistry, vol. 69, pp. 497-529, 2000.

[2] K. Bebenek, C. M. Joyce, M. P. Fitzgerald, and T. A. Kunkel, "The fidelity of DNA synthesis catalyzed by derivatives of Escherichia coli DNA polymerase I," Journal of Biological Chemistry, vol. 265, no. 23, pp. 13878-13887, 1990.

[3] A. Kornberg, DNA Replication, W. H. Freeman, San Francisco, Calif, USA, 1980.

[4] P. K. Gupta, D. L. Johnson, T. M. Reid, M.-S. Lee, L. J. Romano, and C. M. King, "Mutagenesis by single site-specific arylamine-DNA adducts. Induction of mutations at multiple sites," Journal of Biological Chemistry, vol. 264, no. 33, pp. 20120-20130, 1989.

[5] S. Shibutani and A. P. Grollman, "Nucleotide misincorporation on DNA templates containing $\mathrm{N}$-(deoxyguanosin-N2yl)-2-(acetylamino)fluorene," Chemical Research in Toxicology, vol. 6, no. 6, pp. 819-824, 1993.

[6] E. F. Pettersen, T. D. Goddard, C. C. Huang et al., "UCSF Chimera-a visualization system for exploratory research and analysis," Journal of Computational Chemistry, vol. 25, no. 13, pp. 1605-1612, 2004.

[7] S. Korolev, M. Nayal, W. M. Barnes, E. Di Cera, and G. Waksman, "Crystal structure of the large fragment of Thermus aquaticus DNA polymerase I at 2.5 - $\AA$ resolution: structural basis for thermostability," Proceedings of the National Academy of Sciences of the United States of America, vol. 92, no. 20, pp. 9264-9268, 1995.

[8] Y. Li, S. Korolev, and G. Waksman, "Crystal structures of open and closed forms of binary and ternary complexes of the large fragment of Thermus aquaticus DNA polymerase I: structural basis for nucleotide incorporation," EMBO Journal, vol. 17, no. 24, pp. 7514-7525, 1998.

[9] V. Derbyshire, N. D. F. Grindley, and C. M. Joyce, "The 3'-5' exonuclease of DNA polymerase I of Escherichia coli: 
contribution of each amino acid at the active site to the reaction," EMBO Journal, vol. 10, no. 1, pp. 17-24, 1991.

[10] D. L. Ollis, P. Brick, R. Hamlin, N. G. Xuong, and T. A. Steitz, "Structure of large fragment of Escherichia coli DNA polymerase I complexed with dTMP," Nature, vol. 313, no. 6005, pp. 762-766, 1985.

[11] L. S. Beese, V. Derbyshire, and T. A. Steitz, "Structure of DNA polymerase I Klenow fragment bound to duplex DNA," Science, vol. 260, no. 5106, pp. 352-355, 1993.

[12] S. Basu, A. Basu, and M. J. Modak, "Pyridoxal 5'-phosphate mediated inactivation of Escherichia coli DNA polymerase I: identification of lysine-635 as an essential residue for the processive mode of DNA synthesis," Biochemistry, vol. 27, no. 18, pp. 6710-6716, 1988.

[13] J. R. Kiefer, C. Mao, J. C. Braman, and L. S. Beese, "Visualizing DNA replication in a catalytically active Bacillus DNA polymerase crystal," Nature, vol. 391, no. 6664, pp. 304-307, 1998.

[14] S. Doublié, S. Tabor, A. M. Long, C. C. Richardson, and T. Ellenberger, "Crystal structure of a bacteriophage T7 DNA replication complex at $2.2 \AA$ resolution," Nature, vol. 391, no. 6664, pp. 251-258, 1998.

[15] H. Pelletier, M. R. Sawaya, A. Kumar, S. H. Wilson, and J. Kraut, "Structures of ternary complexes of rat DNA polymerase $\beta$, a DNA template-primer, and ddCTP," Science, vol. 264, no. 5167, pp. 1891-1903, 1994.

[16] Y. Li, Y. Kong, S. Korolev, and G. Waksman, "Crystal structures of the Klenow fragment of Thermus aquaticus DNA polymerase I complexed with deoxyribonucleoside triphosphates," Protein Science, vol. 7, no. 5, pp. 1116-1123, 1998.

[17] L. Dzantiev and L. J. Romano, "A conformational change in E. coli DNA polymerase I (Klenow fragment) is induced in the presence of a dNTP complementary to the template base in the active site," Biochemistry, vol. 39, no. 2, pp. 356-361, 2000.

[18] S. Doublié, M. R. Sawaya, and T. Ellenberger, "An open and closed case for all polymerases," Structure, vol. 7, no. 2, pp. R31-R35, 1999.

[19] S. J. Johnson, J. S. Taylor, and L. S. Beese, "Processive DNA synthesis observed in a polymerase crystal suggests a mechanism for the prevention of frameshift mutations," Proceedings of the National Academy of Sciences of the United States of America, vol. 100, no. 7, pp. 3895-3900, 2003.

[20] C. M. Joyce, O. Potapova, A. M. DeLucia, X. Huang, V. P. Basu, and N. D. F. Grindley, "Fingers-closing and other rapid conformational changes in DNA polymerase I (Klenow fragment) and their role in nucleotide selectivity," Biochemistry, vol. 47, no. 23, pp. 6103-6116, 2008.

[21] M. Astatke, K. Ng, N. D. F. Grindley, and C. M. Joyce, "A single side chain prevents Escherichia coli DNA polymerase I (Klenow fragment) from incorporating ribonucleotides," Proceedings of the National Academy of Sciences of the United States of America, vol. 95, no. 7, pp. 3402-3407, 1998.

[22] R. H. Heflich and R. E. Neft, "Genetic toxicity of 2acetylaminofluorene, 2-aminofluorene and some of their metabolites and model metabolites," Mutation Research, vol. 318, no. 2, pp. 73-174, 1994.

[23] S. F. O'Handley, D. G. Sanford, and R. Xu, "Structural characterization of an N-acetyl-2-aminofluorene (AAF) modified dna oligomer by NMR, energy minimization, and molecular dynamics," Biochemistry, vol. 32, no. 10, pp. 2481-2497, 1993.

[24] L. M. Eckel and T. R. Krugh, "2-aminofluorene modified DNA duplex exists in two interchangeable conformations," Nature Structural Biology, vol. 1, no. 2, pp. 89-94, 1994.
[25] L. M. Eckel and T. R. Krugh, "Structural characterization of two interchangeable conformations of a 2-aminofluorenemodified DNA oligomer by NMR and energy minimization," Biochemistry, vol. 33, no. 46, pp. 13611-13624, 1994.

[26] L. Dzantiev and L. J. Romano, "Interaction of Escherichia coli DNA polymerase I (Klenow fragment) with primer-templates containing $\mathrm{N}$-acetyl-2-aminofluorene or N-2-aminofluorene adducts in the active site," Journal of Biological Chemistry, vol. 274, no. 6, pp. 3279-3284, 1999.

[27] O. Rechkoblit, A. Kolbanovskiy, L. Malinina, N. E. Geacintov, S. Broyde, and D. J. Patel, "Mechanism of error-free and semitargeted mutagenic bypass of an aromatic amine lesion by Y-family polymerase Dpo4," Nature Structural and Molecular Biology, vol. 17, no. 3, pp. 379-388, 2010.

[28] H. Miller and A. P. Grollman, "Kinetics of DNA polymerase I (Klenow fragment exo-) activity on damaged dna templates: effect of proximal and distal template damage on DNA synthesis," Biochemistry, vol. 36, no. 49, pp. 15336-15342, 1997.

[29] L. Dzantiev and L. J. Romano, "Differential effects of N-acetyl2-aminofluorene and N-2-aminofluorene adducts on the conformational change in the structure of DNA polymerase I (Klenow fragment)," Biochemistry, vol. 39, no. 17, pp. 5139$5145,2000$.

[30] S. Lone and L. J. Romano, "Mechanistic insights into replication across from bulky DNA adducts: a mutant polymerase I allows an $\mathrm{N}$-acetyl-2-aminofluorene adduct to be accommodated during DNA synthesis," Biochemistry, vol. 42, no. 13, pp. 3826-3834, 2003.

[31] J. B. Bell, K. A. Eckert, C. M. Joyce, and T. A. Kunkel, "Base miscoding and strand misalignment errors by mutator Klenow polymerases with amino acid substitutions at tyrosine 766 in the O helix of the fingers subdomain," Journal of Biological Chemistry, vol. 272, no. 11, pp. 7345-7351, 1997.

[32] S. Dutta, Y. Li, D. Johnson et al., "Crystal structures of 2acetylaminofluorene and 2-aminofluorene in complex with T7 DNA polymerase reveal mechanisms of mutagenesis," Proceedings of the National Academy of Sciences of the United States of America, vol. 101, no. 46, pp. 16186-16191, 2004.

[33] J. Trincao, R. E. Johnson, C. R. Escalante, S. Prakash, L. Prakash, and A. K. Aggarwal, "Structure of the Catalytic Core of S. cerevisiae DNA polymerase $\eta$ : implications for translesion DNA synthesis," Molecular Cell, vol. 8, no. 2, pp. 417-426, 2001.

[34] B. Singer and D. Grunberger, Molecular Biology of Mutagens and Carcinogens, Plenum Press, New York, NY, USA, 1983.

[35] G. W. Hsu, J. R. Kiefer, D. Burnouf, O. J. Becherel, R. P. P. Fuchs, and L. S. Beese, "Observing translesion synthesis of an aromatic amine DNA adduct by a high-fidelity DNA polymerase," Journal of Biological Chemistry, vol. 279, no. 48, pp. 50280-50285, 2004.

[36] R. P. Fuchs and S. Fujii, "Translesion synthesis in Escherichia coli: lessons from the NarI mutation hot spot," DNA Repair, vol. 6, no. 7, pp. 1032-1041, 2007.

[37] N. Koffel-Schwartz, J.-M. Verdier, M. Bichara, et al., "Carcinogen-induced mutation spectrum in wild-type, uvrA and umuC strains of Escherichia coli. Strain specificity and mutation-prone sequences," Journal of Molecular Biology, vol. 177, no. 1, pp. 33-51, 1984.

[38] R. S. Tebbs and L. J. Romano, "Mutagenesis at a sitespecifically modified NarI sequence by acetylated and deacetylated aminofluorence adducts," Biochemistry, vol. 33, no. 30, pp. 8998-9006, 1994. 
[39] J. P. Gill and L. J. Romano, "Mechanism for N-acetyl2-aminofluorene-induced frameshift mutagenesis by Escherichia coli DNA polymerase I (Klenow fragment)," Biochemistry, vol. 44, no. 46, pp. 15387-15395, 2005.

[40] E. C. Friedberg, A. R. Lehmann, and R. P. P. Fuchs, “Trading places: how do DNA polymerases switch during translesion DNA synthesis?" Molecular Cell, vol. 18, no. 5, pp. 499-505, 2005.

[41] M. T. Washington, L. Prakash, and S. Prakash, "Yeast DNA polymerase $\eta$ utilizes an induced-fit mechanism of nucleotide incorporation," Cell, vol. 107, no. 7, pp. 917-927, 2001.

[42] S. Prakash, R. E. Johnson, and L. Prakash, "Eukaryotic translesion synthesis DNA polymerases: specificity of structure and function," Annual Review of Biochemistry, vol. 74, pp. 317-353, 2005.

[43] R. E. Johnson, S. Prakash, and L. Prakash, "Efficient bypass of a thymine-thymine dimer by yeast DNA polymerase, Pol $\eta$," Science, vol. 283, no. 5404, pp. 1001-1004, 1999.

[44] T. Matsuda, K. Bebenek, C. Masultanl, F. Hanaoka, and T. A. Kunkel, "Low fidelity DNA synthesis by human DNA polymerase- $\eta$," Nature, vol. 404, no. 6781, pp. 1011-1013, 2000.

[45] M. T. Washington, R. E. Johnson, S. Prakash, and L. Prakash, "Fidelity and processivity of Saccharomyces cerevisiae DNA polymerase $\eta$," Journal of Biological Chemistry, vol. 274, no. 52, pp. 36835-36838, 1999.

[46] M. T. Washington, R. E. Johnson, S. Prakash, and L. Prakash, "Accuracy of thymine-thymine dimer bypass by Saccharomyces cerevisiae DNA polymerase $\eta$," Proceedings of the National Academy of Sciences of the United States of America, vol. 97, no. 7, pp. 3094-3099, 2000.

[47] C. Xu, B. A. Maxwell, J. A. Brown, L. Zhang, and Z. Suo, "Global conformational dynamics of a Y-family DNA polymerase during catalysis," PLoS Biology, vol. 7, no. 10, Article ID e1000225, 2009.

[48] V. Vooradi and L. J. Romano, "Effect of N-2acetylaminofluorene and 2-aminofluorene adducts on DNA binding and synthesis by yeast DNA polymerase $\eta$," Biochemistry, vol. 48, no. 19, pp. 4209-4216, 2009.

[49] J. Cramer and T. Restle, "Pre-steady-state kinetic characterization of the DinB homologue DNA polymerase of Sulfolobus solfataricus," Journal of Biological Chemistry, vol. 280, no. 49, pp. 40552-40558, 2005.

[50] T. D. Christian, L. J. Romano, and D. Rueda, "Single-molecule measurements of synthesis by DNA polymerase with base-pair resolution," Proceedings of the National Academy of Sciences of the United States of America, vol. 106, no. 50, pp. 21109-21114, 2009.

[51] Y. Santoso, C. M. Joyce, O. Potapova et al., "Conformational transitions in DNA polymerase I revealed by single-molecule FRET," Proceedings of the National Academy of Sciences of the United States of America, vol. 107, no. 2, pp. 715-720, 2010. 

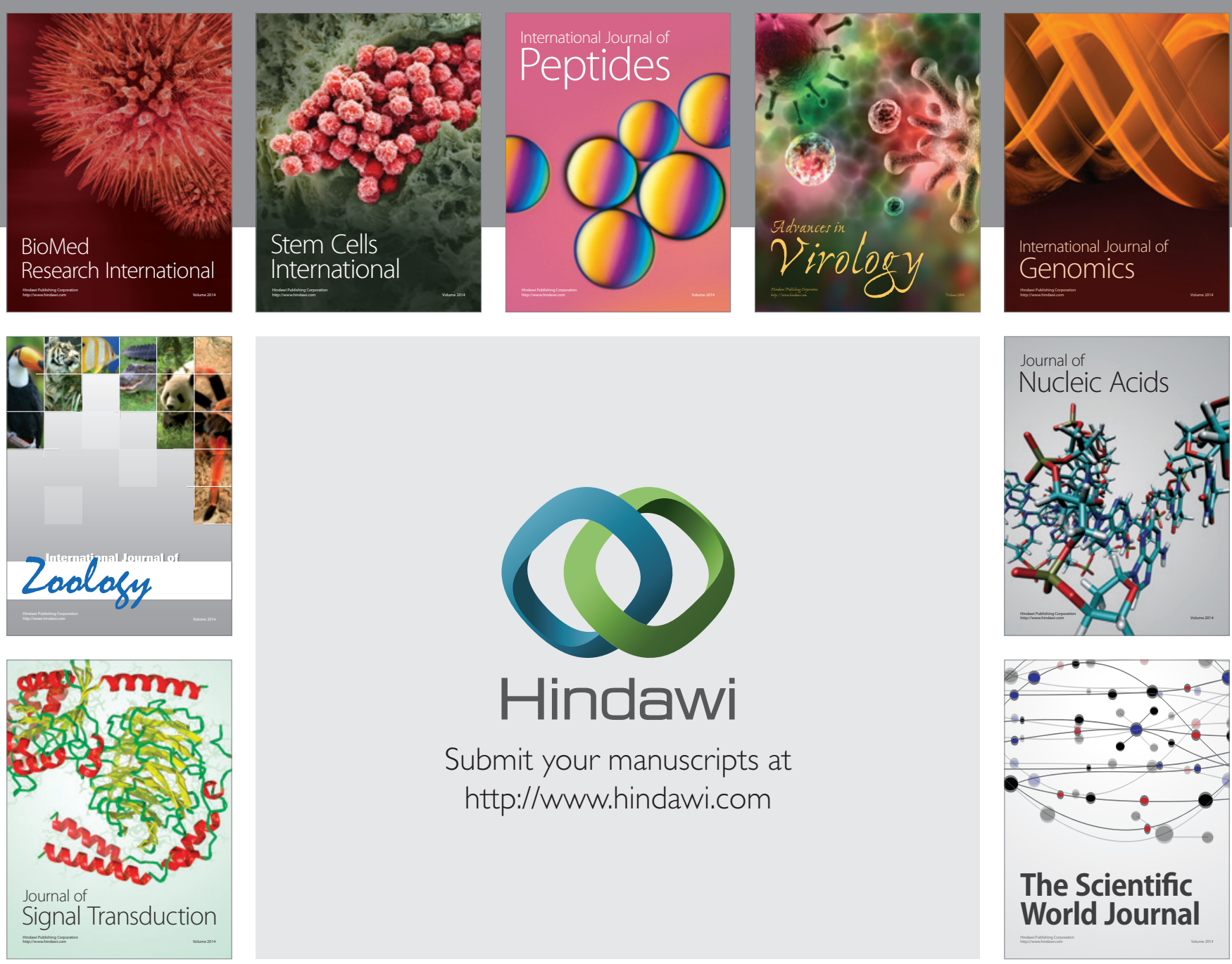

Submit your manuscripts at

http://www.hindawi.com
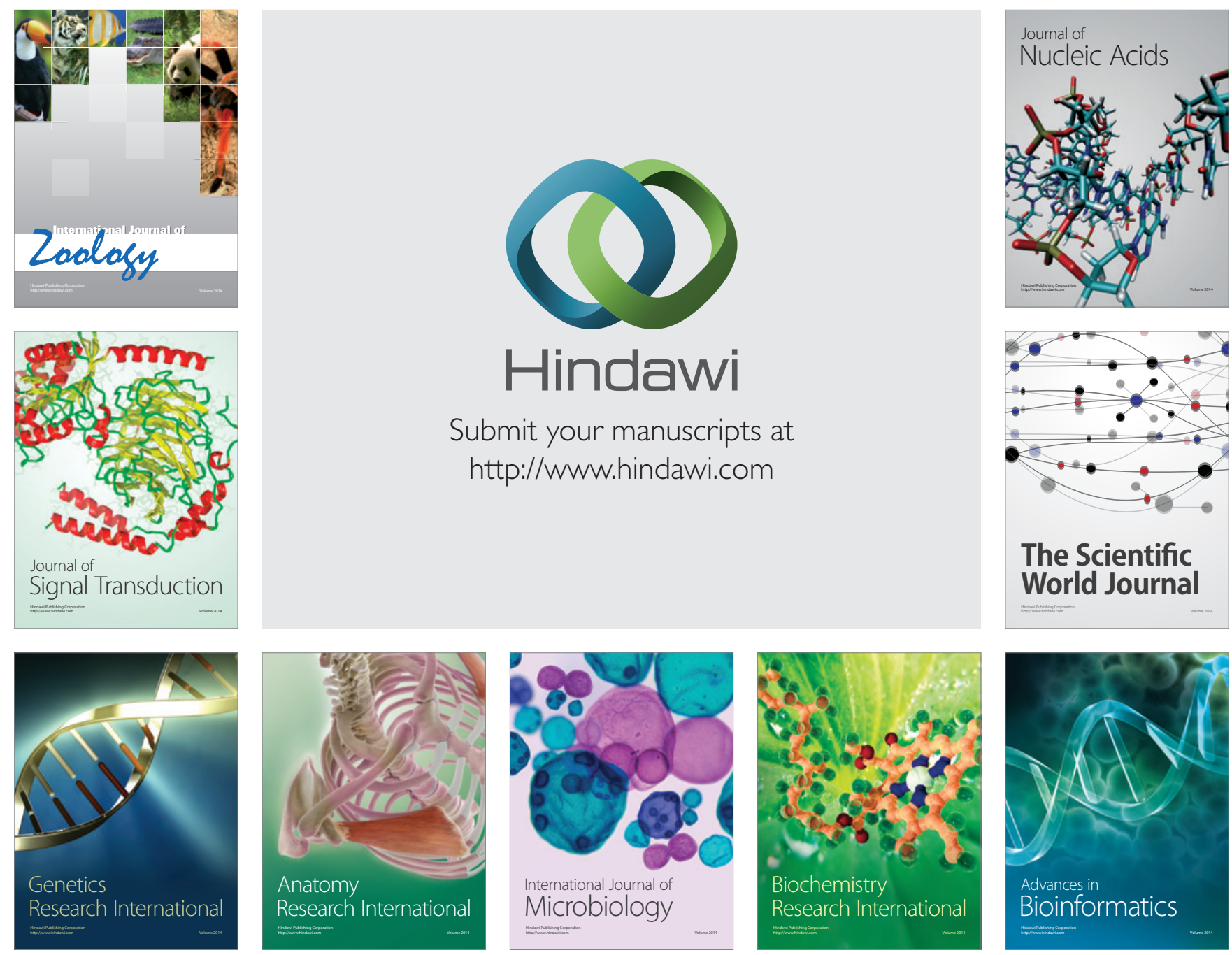

The Scientific World Journal
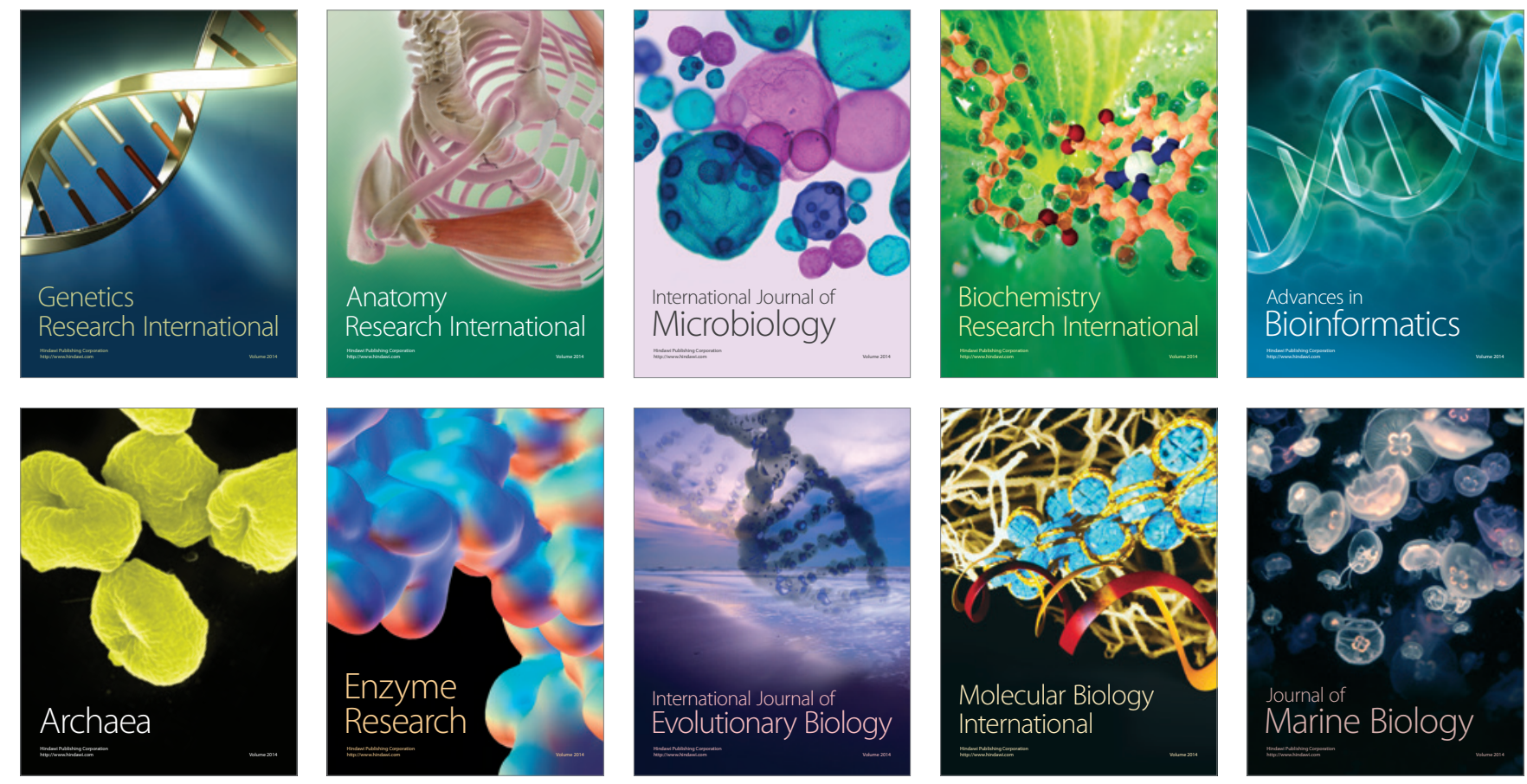